\title{
Fulminant Guillain-Barre Syndrome Mimicking Clinical Brain Death: A Rare Condition With Bad Outcomes
}

\author{
Devika Prasanna $^{\mathrm{a}}$, Nidhi Madan ${ }^{\mathrm{a}}$, Sumit Kapoor ${ }^{\mathrm{a}, \mathrm{b}}$
}

\begin{abstract}
Guillain-Barre syndrome (GBS) is a disease of the peripheral nervous system characterized by acute areflexic paralysis and albuminocytological dissociation on cerebrospinal fluid (CSF). Fulminant GBS mimicking clinical brain death has been described in the literature and has overall poor prognosis since patients either die or are left with severe disability. We describe a case of fulminant GBS mimicking clinical brain death where patient died of unexplained asystolic cardiac arrest related to severe dysautonomia. Dysautonomia is a marker of poor prognosis in this patient population.
\end{abstract}

Keywords: Dysautonomia; Albuminocytological; Quadriparesis; Areflexia; Plasmapheresis

\section{Introduction}

Guillain-Barre syndrome (GBS) is characterized by acute areflexic paralysis and albuminocytological dissociation on cerebrospinal fluid (CSF). Fulminant GBS mimicking clinical brain death has been described in the literature and has overall poor prognosis [1-7]. We describe a case of fulminant GBS mimicking clinical brain death where patient died of unexplained cardiac arrest related to severe dysautonomia.

\section{Case Report}

A healthy 44-year-old male with no significant medical history presented to our hospital's emergency department with complaints of acute shortness of breath requiring initiation of mechanical ventilation. He also gave history of vomiting, diar-

Manuscript accepted for publication May 19, 2015

aDepartment of Medicine, Jacobi Medical Center, Bronx, NY, USA ${ }^{b}$ Corresponding Author: Sumit Kapoor, Division of Pulmonary/Critical Care, Department of Medicine, Jacobi Medical Center, 1400, Pelham Parkway South, Bronx, NY 10461, USA. Email: drkapoorsumit@gmail.com

doi: http://dx.doi.org/10.14740/jmc2181w rhea and fever of 5 days duration. His neurological exam was consistent with severe flaccid quadriparesis and complete areflexia in all four extremities but intact extraocular movements and pupillary anisocoria. Computerized tomography (CT) scan of the head was unremarkable. On day 2 of ICU stay, his neurological exam became consistent with clinical brain death as he developed coma, bilaterally fixed and dilated pupils and loss of extraocular movements. He was not triggering the ventilator. Initial lumbar puncture (LP) was non-confirmatory but repeat cerebrospinal fluid analysis done 1 month later showed albuminocytological dissociation (Table 1). Electromyographic (EMG) study showed findings consistent with primary demyelinating neuropathy with secondary axonal degeneration. Electroencephalographic (EEG) study demonstrated intact cortical function with patient in persistently awake state. His ICU course was complicated by episodes of severe dysautonomia, extremes of blood pressure and symptomatic bradycardia requiring insertion of temporary pacemaker. He received one course of plasmapheresis and two courses of IV immunoglobulin with no clinical recovery. He underwent tracheostomy and gastrostomy tube placement and died about 6 weeks later after developing sudden unexplained asystolic cardiac arrest related to his severe dysautonomia.

\section{Discussion}

GBS, first described in 1916, is an immunologically mediated disease of the peripheral nervous system, characterized by acute areflexic paralysis with albuminocytological disso-

Table 1. Cerebrospinal Fluid Analysis Results

\begin{tabular}{lll} 
CSF studies & On admission & One month later \\
\hline Appearance & Clear & Turbid \\
Color & Colorless & Pink \\
RBCs & None & 180 \\
WBCs & None & None \\
CSF glucose & 79 & 62 \\
CSF total protein & 26.4 & 86 \\
$\begin{array}{l}\text { Albuminocytological } \\
\text { dissociation }\end{array}$ & Absent & Present \\
\hline
\end{tabular}


ciation (high protein levels and normal cell counts) on CSF analysis [8]. It is the most common cause of acute flaccid paralysis worldwide since the eradication of poliomyelitis, with slight male predominance. Up to $20 \%$ of the patients remain severely disabled and about $5 \%$ die, despite immunotherapy [8]. Our patient presented with diarrhea and up to two-thirds of cases are preceded by symptoms of upper respiratory tract infection or diarrhea [8]. Thirty percent of infections have been attributed to campylobacter jejuni infection. Various forms of GBS have been described including typical acute inflammatory demyelinating polyneuropathy (AIDP), acute motor axonal neuropathy (AMAN), acute motor and sensory axonal neuropathy (AMSAN) and Miller-Fisher variant.

Serious and fatal autonomic dysfunction, like arrhythmias and extreme hypertension or hypotension, has been well described in patients with GBS [9]. Three to eight percent of patients with GBS die from complications like sepsis, acute respiratory distress syndrome (ARDS), pulmonary embolism, or in rare cases, unexplained cardiac arrest, related to dysautonomia [8]. Sixty-five percent of patients are left with persistent minor deficits, $20 \%$ with serious disability and only $20 \%$ with no residual problems [8].

Our patient developed fulminant GBS and had clinical exam resembling brain death. Our differential diagnosis included tick paralysis, botulism, brainstem cerebrovascular accident, brainstem encephalitis or fulminant axonal neuropathy. We did not perform any apnea test since patient was not brain dead. Brain death is defined as an irreversible cessation of all functions of the entire brain, including brain stem. There should be an underlying etiology leading to brain death and all reversible causes should be ruled out first. Our patient's EEG also showed activity consistent with persistently awake state, thus ruling out brain death. The patient had albuminocytological dissociation on second CSF sample. Albuminocytological dissociation on CSF was reported in nine out of 12 cases of fulminant GBS mimicking brain death in series by Vargas et al [1]. EEG typically shows presence of alpha rhythm which is unresponsive to painful and auditory stimulation or preserved sleep patterns or reactivity to sound. EMG studies show inexcitability of all nerves, due to distal pathology of the motor axons, either distal conduction block or axonal degeneration. Severe axonal degeneration in GBS is associated with poor clinical outcomes whether the axonal damage is a primary event or is secondary to inflammatory demyelination [5]. Our patient had EMG consistent with axonal degeneration.

GBS mimicking clinical brain death is rare occurrence and about 20 cases have been reported in the literature. This condition has a poor recovery rate and a high mortality, particularly, related to dysautonomia [1]. Outcomes of this subset of patients have been described by Vargas et al in the largest case series of 13 patients in 2000 . Of the three deaths, two died from cardiac arrest due to dysautonomia and one died due to large anterior wall MI. Only two patients recovered with minor deficits and rest were severely disabled [1]. We report, to the best of our knowledge, the third case of death related to dysautonomia leading to sudden explained asystolic cardiac arrest. Nithyashree et al reported that markers of poor prog- nosis in fulminant GBS included elderly patients with a mean age of 60 years, prior diarrheal prodrome, rapid progression of muscle weakness requiring mechanical ventilation within $24 \mathrm{~h}$, dense weakness of all four limbs with cardiovascular autonomic symptoms and inexcitable peripheral nerves [10]. Our patient had most of these features excluding age. Ahmed et al reported a case of young patient with GBS masquerading as brain death who recovered clinically after receiving mechanical ventilation for 150 days [5]. Bernard et al described a patient with fulminant GBS mimicking brain death who died from nosocomial infection after 158 days without any motor recovery [2].

\section{Conclusion}

Fulminant GBS mimicking clinical brain death is associated with poor prognosis as most of the patients either die or are left with severe disability. Dysautonomia is a poor prognostic sign and a marker of mortality in this patient population.

\section{References}

1. Vargas F, Hilbert G, Gruson D, Valentino R, GbikpiBenissan G, Cardinaud JP. Fulminant Guillain-Barre syndrome mimicking cerebral death: case report and literature review. Intensive Care Med. 2000;26(5):623-627.

2. Bernard V, Van Pesch V, Hantson P. Guillain-Barre syndrome mimicking brain death pattern: a poorly reversible condition. Acta Neurol Belg. 2010;110(1):93-96.

3. Kang BH, Kim KK. Fulminant guillain-barre syndrome mimicking cerebral death following acute viral hepatitis a. J Clin Neurol. 2007;3(2):105-107.

4. Joshi MC, Azim A, Gupta GL, Poddar BP, Baronia AK, Singh RK. Guillain-Barre syndrome with absent brainstem reflexes--a report of two cases. Anaesth Intensive Care. 2008;36(6):867-869.

5. Ahmed et al. Guillain-Barre Syndrome Masquerading as Brain Death. Bangla-desh Crit Care J. 2013;1:53-55.

6. Rivas S, Douds GL, Ostdahl RH, Harbaugh KS. Fulminant Guillain-Barre syn-drome after closed head injury: a potentially reversible cause of an ominous exami-nation. Case report. J Neurosurg. 2008;108(3):595-600.

7. Rigamonti A, Basso F, Stanzani L, Agostoni E, Lauria G. Guillain-Barre syn-drome mimicking brain death. J Peripher Nerv Syst. 2009;14(4):316-319.

8. Yuki N, Hartung HP. Guillain-Barre syndrome. N Engl J Med. 2012;366(24):2294-2304.

9. Patel MB, Goyal SK, Punnam SR, Pandya K, Khetarpal V, Thakur RK. Guillain-Barre Syndrome with asystole requiring permanent pacemaker: a case report. J Med Case Rep. 2009;3:5.

10. Nithyashree N, Dhanaraj M, Kumar S, Saraswathi MB. Factors predicting poor outcome in patients with fulminant Guillaine-Barre syndrome. Ann Indian Acad Neurol. 2014;17(4):463-465. 